Topics

\title{
Overview of Elemental Distributions on the Moon Observed by SELENE GRS
}

\author{
By Nobuyuki HASEBE ${ }^{1)}$, Naoyuki YAmashita ${ }^{1)}$, Yuzuru KAROuJI ${ }^{1}$, Shingo KOBAYASHI ${ }^{1)}$, Makoto HAREYAMA ${ }^{1)}$, \\ Kanako HaYATsu $^{1)}$, Shinpei Nemoto ${ }^{1)}$, Kazuya IwABuchi ${ }^{1)}$, Yuko TAKedA ${ }^{1)}$, Hiroshi NagaokA ${ }^{1)}$, Koichi TsukadA ${ }^{1)}$, \\ Osamu OKUDAIRA ${ }^{1)}$, Kunitomo SAKURAI ${ }^{1)}$, Shinichi KOMATSU ${ }^{1)}$, Eido ShibamurA ${ }^{2)}$, Masanori KobaYASHI ${ }^{3)}$, \\ Mitsuru EBIHARA ${ }^{4)}$, Takeshi HiharA ${ }^{4)}$, Tomoko ArAi ${ }^{5)}$, Takamitsu SugIHARA ${ }^{6}$, Hiroshi TAKedA ${ }^{7)}$, Claude d'UstoN $^{8)}$, \\ Olivier GASNAULT ${ }^{8)}$, Benedicte DIEZ ${ }^{8)}$, Olivier FORNI ${ }^{8)}$, Sylvestre MAURICE ${ }^{8)}$, Robert C. REEDY ${ }^{9)}$ and Kyeong Ja KIM ${ }^{10)}$

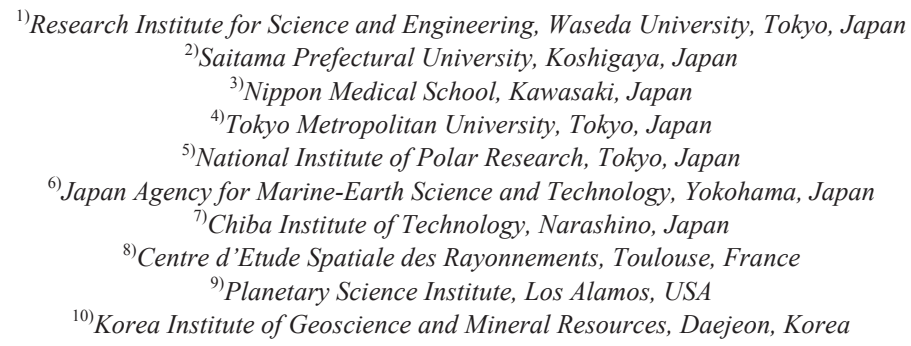

(Received August 18th, 2009)

\begin{abstract}
The high precision gamma-ray spectrometer (GRS) is carried on the first Japan's large-scaled lunar explorer, SELENE (KAGUYA) circling a polar orbit. The GRS consists of a large Ge crystal as a main detector and massive bismuth germanate crystals and a plastic scintillator as anticoincidence detectors. Since the successful launch on Sep. 14,2007 , radiation damage in the Ge detector has been induced from the incidence of energetic particles from space, which degraded its energy resolution. Then the Ge detector was annealed for 2 days at $85 \pm 5 \mathrm{~K}$ and the resolution is recovered to the level at the initial phase of the early observation. The special operations were conducted in December, 2008 in order to measure backgrounds gamma rays from the materials of the spacecraft body and the GRS instrument itself. The GRS data from the $100 \mathrm{~km}$ altitude reveal the global distribution of trace elements of Th and $\mathrm{K}$ on the Moon which delineates the distributions of KREEP component of lunar materials. Th and K-rich materials are concentrated around the Imbrium basin in Procellarum KREEP Terrane (PKT) and intermediately concentrated in South Pole-Aitken basin area on the farside. Results from special operations and observation are described and discussed.
\end{abstract}

Key Words: Moon, Composition, Gamma, Germanium

\section{Introduction}

The measurement of gamma rays coming from the Moon is a powerful method to infer the composition of the matter in the top few tens of centimeters of the surface. When gamma rays are remotely measured from a spacecraft in orbit, gamma ray line energies identify nuclides from which they were emitted, and their fluxes are closely related to their elemental concentration. Energy resolution severely affects the scientific outcome and its quality achieved by gamma-ray measurements. Previous lunar missions, Apollo and Lunar Prospector ${ }^{1)}$, employed scintillation gamma-ray detectors, $\mathrm{NaI}(\mathrm{Tl})$ and BGO with limited energy resolutions, respectively. A gamma-ray spectrometer with excellent energy resolution is highly required for the complex gamma-fields such as the surface of the Moon.

In the first Japanese lunar mission SELENE (KAGUYA) successfully launched from Tanegashima Space Center on Sep. $14,2007^{2)}$, a Ge detector is firstly used as a main detector of its Gamma Ray Spectrometer (GRS) to observe the lunar surface because of its excellent energy resolution ${ }^{3}$. The GRS consists of a large Ge crystal as a main detector and massive bismuth germanate crystals (BGO) and a plastic scintillator as anticoincidence detectors. The GRS has been providing precise global abundances of natural radioactive nuclides such as $\mathrm{K}$, Th and $\mathrm{U}$, and major elements such as $\mathrm{O}, \mathrm{Mg}, \mathrm{Al}, \mathrm{Si}, \mathrm{Ca}$, $\mathrm{Ti}$ and $\mathrm{Fe}$ in the lunar subsurface by remote sensing and the precious data for the future utilization of lunar resources ${ }^{4)}$. In this paper, the special operations of the measurement of background gamma rays, annealing the Ge crystal conducted December, 2008, and recent results of the GRS observation are presented and discussed.

\section{Regular Observation}

From the end of December 2007, the GRS observed lunar gamma rays at a polar orbit of $100 \mathrm{~km}$ in average altitude for 10 months as a nominal mission. After the nominal mission, an extended mission followed. The energy resolution of the GRS was $3 \mathrm{keV}$ for $1.33 \mathrm{MeV}$ gamma rays in pre-flight test and degraded due to radiation damage by high energy cosmic rays. The average energy resolution was about $13 \mathrm{keV}$ for $1.46 \mathrm{MeV}$ gamma rays throughout the nominal mission. $1.33 \mathrm{MeV}$ gamma rays from Cobalt are very weak to detect over the lunar surface. Therefore $1.46 \mathrm{MeV}$ gamma rays from Potassium are used for the energy calibration for in-flight 
condition.

The observation has been made continuously except the interrupt to reset the satellite's reaction wheels accompanied by thruster operations. The interrupt to reset the reaction wheels was 44 minutes at every 12 hours at the begining of nominal mission and was 76 minutes at every 4 hours at the end. The other interrupt was less frequent. In addition, observation was stopped for four months from March 2008 to solve problems in the High Voltage (HV) system of the GRS.

The average dead time in observation was about $20 \%$ due to the processing time of Ge signals and anticoincidence signals from BGO or plastic scintillators. Overall live time in the measurement was approximately 2145 hours in the nominal mission period. Figure 1 shows the global map of the live time accumulated for areas with 30 degrees in latitude and $30 \mathrm{~s}$ in degree longitude. The average, maximum and minimum live times for the $30 \times 30$ degree areas were 29.8, 35.5 and 25.2 hours, respectively.

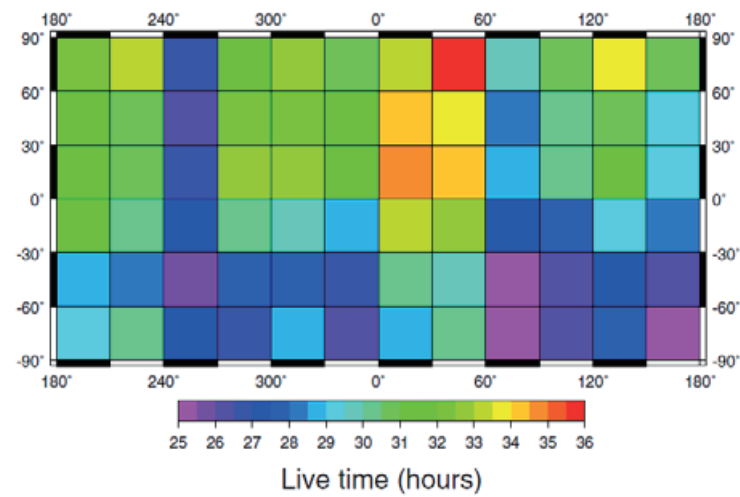

Fig. 1. Accumulation time of the GRS observation in hour binned on $30 \mathrm{x}$ 30 degree area pixels.

\section{Measurement of Background Gamma Rays}

\subsection{Gamma ray spectra}

A gamma-ray spectrum is given by a count rate being expressed as a function of lunar region. Two 8192-channel gamma-ray spectra are accumulated every 17 seconds with an energy resolution of about 0.4 and $1.5 \mathrm{keV}$ per channel $^{3)}$. Long accumulation time is required for our analysis so that many individual spectra have to be summed up. A gamma ray spectrum consists of peaks from line gamma rays above a smooth continuum of counts from many sources, such as Compton scattered gamma rays. The gamma rays from $\mathrm{O}, \mathrm{Si}$, $\mathrm{K}, \mathrm{Ca}, \mathrm{Ti}, \mathrm{Fe}$, Th, and $\mathrm{U}$ appear to mainly come from the Moon, although there are probably local backgrounds from materials of spacecraft and detector itself. The peaks from Al are mostly attributed to the Al structure of the GRS and its surroundings. Many peaks originates from the Ge crystal, including broad "sawtooths" from nuclear interaction of fast neutrons with Ge nuclei. The existence of such wide peaks from Ge shows that the fluxes of fast neutrons in the Kaguya spacecraft are so high that they could also be a strong source of the radiation damage to its GRS.

\subsection{Measurements of background gamma rays}

Some of gamma rays observed by the GRS are both from the Moon and local materials. Careful analyses are needed to accurately retrieve lunar gamma rays. Background measurement of gamma rays and the numerical simulation of gamma and neutron production and their transport in detail are required to improve the precision of the measured chemical abundances. Therefore we made the measurement of background gamma rays for the quantitative evaluation of the lunar surface material. Special spacecraft operations for the measurement were performed twice by controlling the spacecraft's attitude so that the GRS pointed away from the Moon. In these operations, the Ge detector views only deep space instead of looking at the lunar nadir, and accordingly the spacecraft body was located between the Ge detector and the Moon, and behaves as an additional shield.

The first measurement of background gamma rays was carried out in July, 2008, and its result showed that gamma rays from $\mathrm{K}, \mathrm{U}, \mathrm{Ti}, \mathrm{Al}$ and $\mathrm{Mg}$ contained in the spacecraft body and instrument itself were detected at significant magnitude. In particular, the contribution of those gamma rays from Al is significantly large because most structure of the spacecraft and the mission payloads are made of Al. In the second operation of the measurement in December, 2008, gamma ray data were obtained with enough statistics to identify the $1368.6 \mathrm{keV}$ peak from $\mathrm{Mg}^{5}$. Accumulation time of background measurements was approximately 37,000 seconds in total.

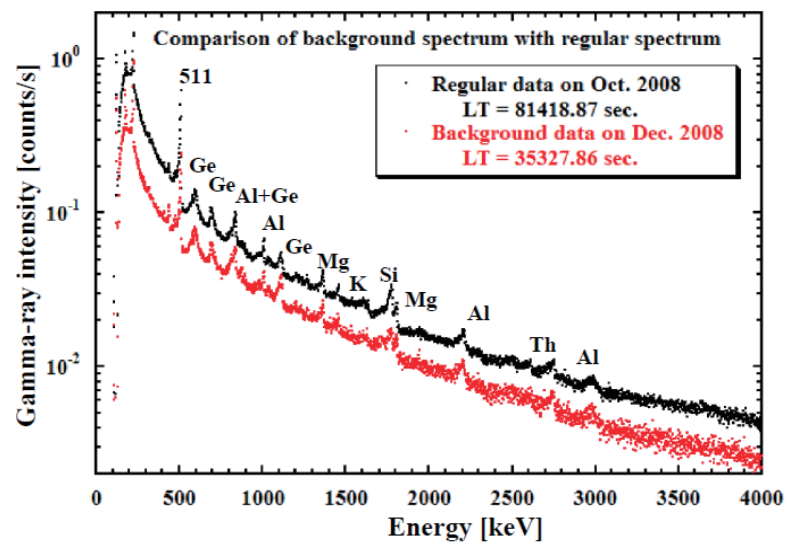

Fig. 2. The background spectrum of gamma-rays observed by special operation when the GRS looks at deep space (bottom). Also shown is the energy spectrum in regular operation when the GRS looks down the lunar nadir (top).

Energy spectra determined from the regular operation and the background operation are presented in Fig. 2. Peaks from $\mathrm{K}, \mathrm{Th}, \mathrm{U}, \mathrm{Ti}, \mathrm{Al}$ and $\mathrm{Mg}$ have been indentified in the background spectrum.

\section{Annealing of the Germanium Detector}

\subsection{Radiation damage in germanium detectors}

Radiation damage is induced to the Ge detector from the incidence of high-energy particles from space. Solar activity from September in 2007 to June in 2009 was so quiet that solar particle events (SPEs) were hardly observed except for a few small ones. Therefore, the dominant source of radiation damage in the Ge detector mainly arises from protons in the 
galactic cosmic rays (GCRs). Helium particles in the GCR and secondary neutrons resulting from intra-nuclear cascades in the lunar material and the detector itself or spacecraft materials also contribute significantly. Displacement effects permanently result in radiation damage to the lattice structure. High energy particles, in particular, produce single defects and extended disordered regions resulting from collisions with $\mathrm{Ge}$ nuclei. The latter is primarily the displacement from the secondary Ge recoils, which can displace thousands of Ge atoms from their equilibrium lattice positions. These disordered regions may be extended up to several hundred microns. As the trapping time becomes longer than the shaping time constant of amplifier, the charges formed by the ionization process in Ge are lost. Therefore, the peaks of line gamma rays in an energy spectrum in a radiation-damaged detector are characterized by a broadening and tailing to the low energy region, and the energy resolution of such Ge detectors degrades. For n-type detectors, the threshold for radiation damage is considered to be about $5 \times 10^{7}$ protons $/ \mathrm{cm}^{2}$ high energy protons and $10^{8}$ neutrons $/ \mathrm{cm}^{2}$ for fast neutron ${ }^{6-8)}$.

If a detector in space is kept colder than about $90 \mathrm{~K}$, this damage is not observed for several years. If the detector is once warmed above this temperature and then cooled to operational temperatures, the damage is prominently observed in the spectra. However, radiation damage can be annealed by heating the detector to high temperatures.

\subsection{Germanium detector in Kaguya GRS}

The lunar exploration was carried out by an intrinsically pure Ge crystal of about $252 \mathrm{~cm}^{3}$ in a closed-end coaxial configuration. The n-type Ge detector was manufactured by Eurisys Mesures and was hermetically encapsulated in a high vacuum-tight $\mathrm{Al}$ canister. The outer implanted $\mathrm{p}+$ contact is $<0.5 \mu \mathrm{m}$ thick. The $\mathrm{n}+$ and $\mathrm{p}+$ contacts are formed by diffusion of lithium and implantation of boron, respectively. The crystal diameter is $65 \mathrm{~mm}$ and its length $77 \mathrm{~mm}$. The signal is extracted from the $\mathrm{n}+$ contact and fed to a charge sensitive preamplifier. It is confirmed that a bias voltage of $+3.1 \mathrm{kV}$ fully depleted the crystal and gave an optimum spectral resolution of $3.0 \mathrm{keV}$ full-width-half maximum (FWHM) at $1332 \mathrm{keV}$ of ${ }^{60} \mathrm{Co}$ standard source at a testing facility in Tsukuba in prior to the launch.

Kaguya GRS started to cool the Ge detector on November 13,2007 , initial check for the GRS was done on November 16, 2007, and regular observation began on December 14, 2007. The typical energy spectrum at the initial and final two weeks of nominal observation mission was shown in Fig. 3. A long tail at lower energy part of the peak is formed by radiation damage. As a result, the tail is well described by an exponential form. The high-energy half of the peak has a Gaussian shape, as expected for a Ge detector with no radiation damage.

The energy resolution has a tendency to degrade with time as shown in Fig. 4. At the end of nominal mission, the energy resolution at $1461 \mathrm{keV}$ is about $21 \mathrm{keV}$ which is seven times larger than that before the launch. It is clear that the spectral performance of the Ge detector degrades with space dose absorbed in orbit. The total fluence of particles from the launch (September 14, 2007) to the initial check for the GRS (November 16, 2007) is estimated to exceed $10^{7}$ particle $/ \mathrm{cm}^{2}$ from the trajectory of Kaguya orbit, which explains the initial resolution of the Ge detector in the nominal mission.

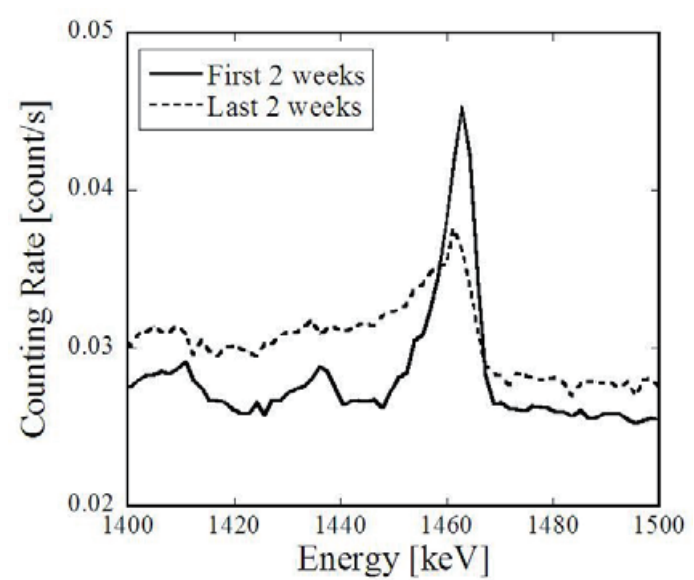

Fig. 3. The comparison of energy spectra of $1461 \mathrm{keV}$ gamma rays in the first and last 2 weeks of the nominal mission.

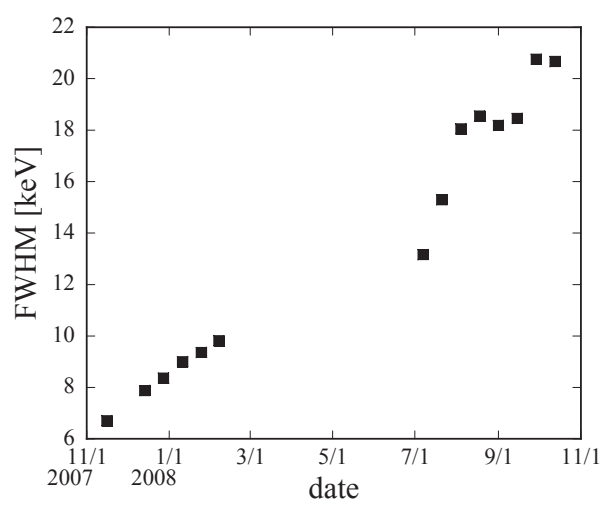

Fig. 4. Temporal variation of the energy resolution for $1461 \mathrm{keV}$ gamma rays thoughout the nominal mission.

Due to other operations of Kaguya spacecraft, cooling of the Ge detector had to be stopped several times as well as the gamma-ray observation. As a result of radiation dose absorbed and rise in temperature, the degradation of detector resolution appeared.

\subsection{Annealing and its results}

The annealing time and temperature are determined from the degree of damage and recovery required. A moderately damaged Ge crystal is expected to be significantly restored by annealing at temperature of about $100{ }^{\circ} \mathrm{C}$ for a period of the order of 1-2 days ${ }^{9}$. For temperatures less than $100{ }^{\circ} \mathrm{C}$, annealing times should be longer ${ }^{10)}$. The annealing temperature in Kaguya GRS was limited by the manufacturer standard to $100{ }^{\circ} \mathrm{C}$.

The detector was held at an intermediate temperature of $20{ }^{\circ} \mathrm{C}$ for $24 \mathrm{~h}$, then warm up to $50{ }^{\circ} \mathrm{C}$ and kept for $24 \mathrm{~h}$, and then heated up to $85 \pm 5{ }^{\circ} \mathrm{C}$ for about 2 days. After annealing, the performance of the Ge detector was checked with $\mathrm{HV}=$ $2.5 \mathrm{kV}$. The energy spectrum after the annealing is shown 
together with that just before the annealing in Fig. 5. It is found that the tails of peaks became much smaller. The energy resolutions have been improved to $7 \mathrm{keV}$, comparable to that at the initial phase of the nominal mission.

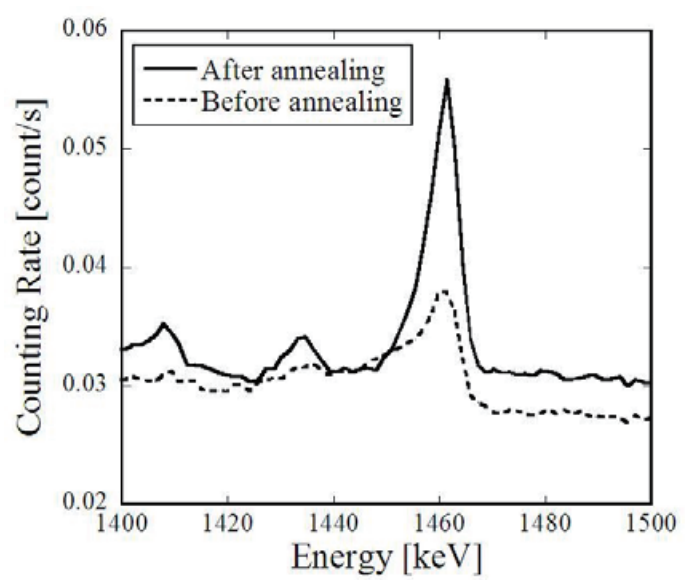

Fig. 5. The comparison of energy spectra of $1461 \mathrm{keV}$ gamma rays observed for 2 weeks before and after annealing the Ge detector.

\section{Global Mapping of Natural Radioactivenuclides}

Gamma-ray spectra containing peaks from various elements are obtained for constraining the geology of the Moon by Kaguya GRS observation. The unique identification of peaks measured by the Ge detector is essential in the complex mixed gamma-ray field in order to precisely derive elemental abundances.

Clear peaks from natural radioisotopes of ${ }^{40} \mathrm{~K}$ and daughters of ${ }^{232} \mathrm{Th}$ and ${ }^{238} \mathrm{U}$ are identified in spectra of gamma rays. These data delineate the global distributions of key trace elements rich in component of lunar materials called KREEP ${ }^{6}$. In Fig. 6, the global distribution of thorium is shown as a typical example. The abundances of the incompatible elements $\mathrm{K}$, Th and $\mathrm{U}$ have considerable regional variations over the lunar surface. As can be seen from Fig. 6, the nearside tends to record higher abundances than the farside. Especially, over the Procellarum KREEP terrain (PKT) is the highest. The South Pole-Aitken terrain (SPAT) also has higher abundances of these elements than the surrounding regions, and the lowest was found in the nearside feldspathic region.

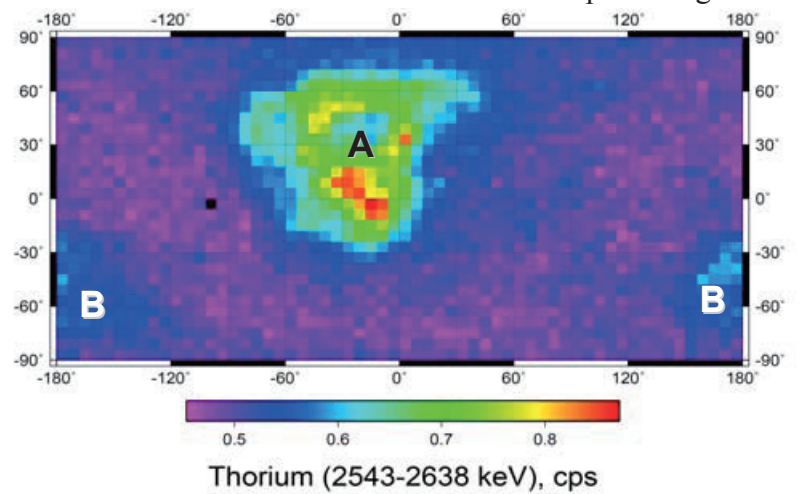

Fig. 6. The global distribution of thorium measured by Kaguya GRS. The symbol of "A" represents PKT region and that of "B" represents SPAT region.
Figure 7 presents the count rate map of potasium measured for the Imbium basin region. Some high-K regions associated with craters near the rim of Imbrium are seen: Aristillus and Mairan etc.. Mountains of Jura, Archimedis and Carpathian are also abundant in $\mathrm{K}$. The wide region from the Carpathian Mountains to Mare Insularum, the North Congnitum, Fra Mauro, and the north Nubium, has very high $\mathrm{K}$ abundance so that this region is identifiable as an ejecta from the Imbrium impact. The $\mathrm{K}$ abundances surrounding Copernicus are asymmetric. Relatively high-K regions also exist near the Aristarchus and highlands north of Imbrium basin which appears to be Imbrium ejecta. There are high-K regions around the Appennine Bench, which seemed to form by post-Imbrium KREEP basalt volcanism ${ }^{11)}$. It should be noted that $\mathrm{K}$ abundance is significantly lower than the surrounding highlands. Coperinicus shows a relatively low abundance of the element $\mathrm{K}$ in comparison with the surrounding highest region, which suggests that the lack of a high-K signature at Copernicus may mean the region happened to be a place where the Imbrium ejecta was lower in $\mathrm{K}$ abundance. Ejecta from a large basin such as Imbrium may be considerably inhomogeneous in spatial extent.

The way how KREEP was formed and distributed is very complex. The observation that the highest surface abundances of natural radioactive nuclides are quite localized around Imbrium basin, which tells us a special Th/U/K-rich terrain on the Moon. The Imprium impact spreads large amounts of natural radioactive material over the Moon. During the period (the post-Imbrium Apennine Bench Formation) of KREEP volcanism, natural radioactive elements were distributed in the regions located around the Apennine Bench, Aristillus and near Sinns Iridum. Following the periods of KREEP volcanism, mare basalt filled in the Imbrium basin with lower amounts of natural radioactive material. And then, additional natural radioactive materials were excavated in various locations around Imbrium basin, when impacts producing the craters Aristarchus, Mairan, and Aristillus pressed out the basalt layer exposing underlying natural radioactive materials $^{11)}$.

In this work, the count rate map of Th over the Moon and the regional $\mathrm{K}$ count rate map are reported. After careful corrections of altitude variation in the orbit of Kaguya spacecraft and background gamma-rays in the energy band of peak area, the global maps of absolute concentration of radioactive nuclides, Th, $\mathrm{U}$ and $\mathrm{K}$ will be presented elsewhere in the near future. Moreover, the abundance of major elements in the lunar surface will also be globally reported after vigorous simulations to support the precise interpretation of observation data. The combination of GRS data with other precise data from other Kaguya instruments such as LISM, LALT, VRAD/RSAT, and LRS will provide new insight about how KREEP was formed and distributed on the lunar surface. 


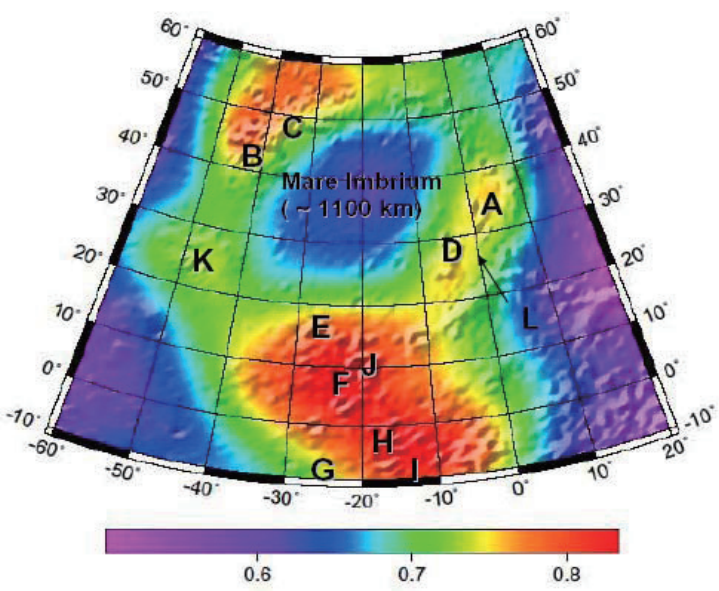

Potassium (1440-1474 keV), cps

Fig. 7. The count rate map of potassium measured for the Imbium basin region. Each symbol represents a moutain or a basin $(\mathrm{A}=$ Aristillus, $\mathrm{B}=$ Mairan, $\mathrm{C}=$ Moutains of Jura, $\mathrm{D}=$ Archimedis, $\mathrm{E}=$ Carpathian Mountains, $\mathrm{F}=$ Mare Insularum, $\mathrm{G}=$ the north Congnitum, $\mathrm{H}=$ Fra Mauro, $\mathrm{I}=$ the north Nubium, $\mathrm{J}=$ Copernicus, $\mathrm{K}=$ Aristarchus, $\mathrm{L}=$ the Appennine Bench).

\section{Acknowledgments}

We would like to thank the staff of JAXA and NEC Toshiba-Space System Ltd. for generous supports in tests of KAGUYA. We also would like to thank KAGUYA-LALT team; the global 1 degree grid topographic data obtained by them is used to produce the shaded relief map of Fig. 7.

\section{References}

1) Lawrence, D. J. et al.: Global Elemental Maps of the Moon, the Lunar Prospector Gamma-Ray Spectrometer, Science, 281 (1998), pp.1484-1489.

2) Kato, M. et al.: The Japanese Lunar Mission SELENE, Science Goals and Present Status, Adv. Space Res., 42 (2008), pp.294-300.

3) Hasebe, N. et al.: Gamma-Ray Spectrometer (GRS) for Lunar Polar Orbiter SELENE, Earth, Planets and Space, 60 (2008), pp.299-312.

4) Hasebe, N.et al.: First Results of High Performance Ge Gamma-Ray Spectrometer onboard Lunar Orbiter SELENE (KAGUYA), J.Phys. Soc. Jpn., 78 (2009), Suppl. A, pp. 18-25.

5) Reedy, R. C., Arnold, J. R. and Trombka, J. I.: Expected $\gamma$ ray Emission Spectra from the Lunar Surface as a Function of Chemical Composition, J. Geophys Res., 78 (1973), pp. 5847-5866.

6) Brückner, J. et al.: Proton-Induced Radiation Damage in Germanium Detectors, IEEE Trans. Nucl. Sci. NS-38 (1991), pp. 209-217.

7) Thomas, H.G. et al.: Neutron Induced Damage of an N-type Ge Detector, Influence of the Operating Temperature, Nucl. Instr. and Meth. A 332 (1993), pp. 215-219.

8) Evans, L.G. et al.: Charged-Particle Induced Radiation Damage of a HP Ge Gamma-Ray Detector during Spaceflight, Nucl. Instr. and Meth. A 422 (1999), pp. 586-590.

9) Pirard, B. et al. : Solar Proton Damase in High-Purity Germanium Detectors, Nucl. Instr. and Meth. A 572 (2007), pp. 698-707.

10) Albernhe, F. et al.: Degradation and recovery of Ge detector: Tests prior to a space mission, Nucl. Instr. and Meth. A 492 (2002), pp. 91-96.

11) Spudis, P.D.: Composition and Origin of the Appennin Bench Formation, Proc. Lunar Planet. Conf. 9 ${ }^{\text {th }}$, (1978), pp.33793394.

12) Lawrence, D. J. et al.: High Resolution Measurement of Absolute Thorium Abundances on the Lunar Surface, Geophys. Res. Letters, 26 (1999), pp. 2681-2684. 